\title{
Convergence as public health innovation: a case for tobacco control
}

This article was published in the following Dove Press journal:

Innovation and Entrepreneurship in Health

21 November 2016

Number of times this article has been viewed

\section{Kimberly A Horn' \\ Maliha Ali' \\ Laurel E Curry' \\ Kenneth P Tercyak ${ }^{2}$ \\ Ray Niaura ${ }^{3}$}

'Department of Prevention and Community Health, The Milken Institute School of Public Health, The George Washington University, ${ }^{2}$ Cancer Prevention and Control Program, Georgetown Lombardi Comprehensive Cancer Center, ${ }^{3}$ Schroeder Institute for Tobacco Research and Policy Studies, Truth Initiative, Washington, DC, USA
Correspondence: Kimberly Horn Department of Prevention and Community Health, The Milken Institute School of Public Health, The George Washington University, $950 \mathrm{New}$ Hampshire Avenue, Washington, DC 20052, USA

Tel +l 2029943180

Email khornI@gwu.edu
Abstract: The tobacco epidemic remains a significant global public health crisis, killing six million people a year. In the US, tobacco use remains the leading cause of preventable death, despite decades of antismoking efforts. Evidence-based tobacco control policies and programs are not consistently or optimally implemented across states and regions, thereby weakening their effectiveness. Moreover, wide disparities persist in tobacco use and tobacco-related diseases among the most socioeconomically vulnerable groups in society. Innovative tobacco control approaches are sorely needed to address these gaps in tobacco control by consolidating and harmonizing siloed and fragmented tobacco control practices. The present review gleans important insights on the nature and potential of this convergence and its applications in public health innovations. The evidence provides important guidance for formulating and implementing strategies for convergence in innovation. The DC Metro Tobacco Research and Instruction Consortium (MeTRIC) provides a case example - describing real-world insights from the authors' experience in implementing convergent innovation. With its two definitive principles in which, 1) public health and development go hand in hand, and 2) a focus on improving the status of the underserved, the concept of convergence leans on innovation to address specific needs of the community. In the multidisciplinary realm of tobacco control, the role of convergent innovation in addressing gaps resistant to current efforts is imperative and required.

Keywords: convergence approach, public health innovation, tobacco control, multisector collaboration

\section{Background Statement of problem}

The tobacco epidemic remains among the most significant public health threats the world has ever faced, killing six million people a year. ${ }^{1}$ In the US, tobacco use persists as the leading cause of preventable death. ${ }^{2}$ An enormous body of conclusive evidence delineates the consequences of tobacco use on human health, particularly combustible tobacco products. ${ }^{2}$

Evidence-based tobacco control policies and programs are not consistently or optimally implemented across states and regions. ${ }^{3}$ This is often due to differences in priorities, policies, and resources available to enact comprehensive public health and tobacco control programs. Unfortunately, across all states and regions, disparities persist in tobacco use and tobacco-related diseases among groups differentiated by race/ethnicity, socioeconomic status, educational attainment, mental health, sexual orientation, occupation, and geography. ${ }^{4}$ These disparities contribute significantly 
to poorer population health outcomes. Innovative tobacco control approaches are needed to accelerate the tempo and scope of public health impact across disparate groups at the population level to achieve health equity. Research is the nexus for innovation and discovery. ${ }^{5}$ Although the US has made tremendous progress in tobacco control since the release of the first US Surgeon General's report in 1964, silos and fragmentation within and outside of academe, the public and private sectors, and communities limit innovation in tobacco control research..$^{6,7}$

\section{Need for innovation in tobacco control}

The word innovation is often used but not well understood or defined. A recent systematic review of public health interventions generated a definition of innovative public health interventions that has utility for this review. ${ }^{8}$ Accordingly,

...Innovative public health interventions (PHIs) are gener-

ally new and different to established interventions. They

should be equitable, applicable to all in a population,

cost-effective and may address health determinants in the

non-health sector of society. ${ }^{8}$

We accept this definition and posit that tobacco control innovations should have several additional features as follows: 1) achieve prevention, abstinence, reductions in consumption, and reductions in harm; 2) provide open and working pathways for innovations to flow to the people and communities for whom the benefits are intended; 3) come in multiple forms, singularly or conjointly, including new ideas, devices, tools, technologies, programs, or methods; and 4) emphasize serving difficult-to-reach individuals and communities who live with the highest burden of health challenge.

Part of the imbalance between tobacco control research and innovation is that a significant portion of research dollars is directed toward traditional sectors such as academia instead of collaborative public-private partnerships, including but not limited to academic researchers. Recently, federal agencies such as the National Institutes of Health, the Centers for Disease Control and Prevention, and Patient-Centered Outcomes Research Institute have established initiatives to more fully engage patients and communities in research..$^{9-11}$ Federal agencies have mechanisms to support small business grants that require a partnership with academic entities. ${ }^{12}$ While these are important and critical steps toward achieving and translating high impact research, we relinquish the full spectrum of innovation potential by not integrating diverse public and private sectors alongside of and within intended communities. More specifically, convergence is not a common research tactic.

\section{The potential of convergence as innovation in tobacco control}

Coalescing multiple sectors to solve problems in innovative ways can be explained by the concept of convergence, ${ }^{13,14} \mathrm{a}$ strategy often overlooked and underimplemented specifically in tobacco control research and more broadly in public health research. Convergence is the transformation and escalation of interactions among different disciplines, technologies, communities, and domains to achieve mutual compatibility and integration of multiple sectors. ${ }^{14}$

Convergence theory adopts a complex "system of systems" approach and is a common feature of numerous theories of social change. ${ }^{15-17}$ Fundamentally, it explains why people and societies migrate toward conditions of similarity. Convergence can occur on numerous levels ranging from the smallest, nanoinformation, to individual and population scales and beyond. Most often, convergence is driven by societal values and needs for progress or improvement. ${ }^{14}$ Convergence provides momentum to an innovation helix ${ }^{14}$ serving as both a propellant and a product; innovation is essential to sustain convergence.

As applied to tobacco control, convergence requires branching out in unconventional and unexpected ways to create added value innovations and to meet shared goals in a mutually serving tobacco control agenda. For the purposes of this review, we define convergence as, the bringing together of academic, public and private sectors, local and regional health authorities, and citizens to develop and implement massive innovative tobacco research initiatives within defined communities. The overarching goal is, through tobacco control, to achieve economic productivity, societal equity and sustainability, and empowerment of individuals and communities, continuing beyond the life of research funding or specific projects. ${ }^{14}$

Convergent approaches for tobacco control innovations may be particularly important among disadvantaged socioeconomic groups where health inequities are greatest and most acute. ${ }^{14,18}$ Globally, there is an "inverse gradient" 19 of tobacco control success, where the tobacco reductions in wealthy environments contrast with increases in tobacco use and tobacco-related illness in poorer environments. ${ }^{19}$ While issues pertaining to unemployment, food insecurity, and environmental stressors are common barriers, low socio-economic status groups are not homogeneous. Yet, this population is consistently most burdened by failures to sustain changes in tobacco use. ${ }^{20}$ One reason could be that existing tobacco control programs have failed to integrate, "converge," and coordinate all relevant channels of influence, 
many of which may be outside of the traditional health and education sectors. ${ }^{21}$ In responding to population-level social needs and values, convergence is an apt strategy against what is sometimes called "the personalization agenda" 22 - when public health efforts focus disproportionately on individual vs. community- or population-level needs. While social ecological models have attempted to consider the myriad levels of influence on health behavior, they fall short on dynamic contextual factors, a feature central to convergent approaches.

Convergent approaches can bridge the disconnect that exists sometimes between research (knowledge) and application by strategically disrupting conventional processes of research investments and distribution of resources. Convergence can also stimulate methods to bypass the lack of a public health infrastructure by linking resources from other sectors with interests in the community. With limited funding options for research, the convergence of public-private sectors alongside communities may be our best bet to yield the knowledge, resources, and technology that is at the core of tobacco control innovation and progress. In addition, in light of competing health priorities, convergence offers a solution to systematically and simultaneously work on multiple priorities (that would be otherwise seen as competing for resources). For instance, the tobacco epidemic disproportionately affects populations that face other health issues such as HIV, substance abuse, and cardiovascular disease.

The idea of convergence strategies is not necessarily new to tobacco control experts. Local coalitions, reflecting diverse perspectives, experiences, cultures, and varying levels of authority, are recognized as popular forces for improving community health, including tobacco control. ${ }^{23,24}$ The convergence perspective, however, takes coalition building further by integrating objectives and activities, sharing fiscal resources, creating policy congruence across organizations (public and private), and capitalizing on varying skill sets offered by converging bodies for a unified purpose. The process is inherently diverse and thereby encourages buy-in from all stakeholders. The exchange of skills and contribution of knowledge across boundaries create a bonded, efficient enterprise. Successful tobacco control requires partnering with other entities with a similar focus on common root causes or problems. ${ }^{25}$ To that end, opportunities for convergence in tobacco control abound since tobacco is a leading risk factor for chronic illness such as cancer and cardiovascular disease. ${ }^{1}$ For example, tobacco control programs can be integrated with programs or entities focused on respiratory illness (such as asthma and tuberculosis) and cardiovascular disease. Interventions to curtail such chronic diseases are incomplete in the absence of a tobacco control element. Such intrafield convergence can spill over from incorporation in primary and dental health care to other areas such as environmental pollution, housing policy, food, employment, security/fire safety, and so on. ${ }^{25}$ Tobacco control requires multisector efforts that merge it into broader health and economic development agendas. The tobacco control community, regardless of where that community resides locally, nationally, or globally, necessitates innovative partnerships beyond conventional boundaries. ${ }^{25}$

Such strategies, however, are often difficult to implement in real-world situations, and the use of convergent partnerships is not widespread. Organizational infrastructure, policies, historical priorities, culture, language, and communication styles are not easily synchronized between entities. This is aptly illustrated in relations between the tobacco control advocates and the tobacco industry. The field of tobacco control has had a clear adversary in the tobacco industry - an industry that has significant influence on international trade. In light of the tobacco industry's reach into philanthropic, scientific, and even academic enterprises, the response of tobacco control advocates has been one of suspicion and exclusion. ${ }^{26,27}$ Viewed from a convergence lens, this is one challenge that the tobacco control field needs to grapple with. The Framework Convention on Tobacco Control (FCTC) is one of the best examples in recent history of an attempt to achieve multisectoral collaboration for large-scale global tobacco control. While the FCTC did converge the public health, economic, development, and political leadership and activities on a global scale, it also, with good reasons, rejected any possibilities of collaborating with the tobacco industry whose products irrefutably cause disease, disability, and death. ${ }^{28}$ More recently, though, there has been accelerated interest in developing safer tobacco and nicotine delivery products and in modern technological advances and appropriate regulatory structures that might permit tobacco users to switch over to these new products. An exclusionary stance precludes the potential meaningful convergence experienced by other major industries impacting public health such as food and alcohol. If this is a bridge too far, tobacco control initiatives that converge with organizations focused on public health problems such as obesity, metabolic diseases, HIV, and substance abuse seem a more plausible and less contentious starting place.

Empirically based collaborative initiatives based on convergence are receiving increased attention in the US and especially in global health care in recent years. ${ }^{18,29}$ This movement is in part due to shrinking budgets in the face of 
expanding health disparities and greater health care demands around the world. ${ }^{23,29}$ Even though a number of studies have examined early implementation of health-related initiatives that center on convergence, less research has been conducted to determine what happens beyond that point of initiation. Moreover, there is limited literature that examines the extent to which convergent approaches are pursued relative to tobacco control, one of the public health's greatest challenges.

To understand the current state of the literature on the utility of convergent approaches in tobacco control, the present study examined what is known or published in this area and identified areas in which further research would be useful to the field. This manuscript reviews tobacco control and other public health programs that feature practices, programs, or interventions involving multisector convergence in the US and globally. We conclude with a case example and recommendations that serve as a practical guide for potential implementations of convergent approaches to tobacco control research.

\section{Methods}

\section{Search terms and review process}

We searched the empirical and gray (nonacademic) literature for reviews and primary research articles published between 2010 and 2016 (May) using a multiple-step Internet search process and predefined search terms that capture our concept of convergence as multisector collaboration. We used PubMed as our primary search engine. While there were no restrictions globally, we included English-language articles only.

First, we searched for literature related to collaborative community-derived tobacco control programs using the following initial search string:

Search 1: tobacco AND (prevention OR control OR program) AND (collaboration OR partnership) AND (community OR state OR city OR county).

Next, we added to the initial search string terms with consideration to innovation and convergence as follows:

Search 2: tobacco AND (prevention OR control OR program) AND (collaboration OR partnership) AND (community OR state OR city OR county) AND (innovation OR convergence*).

Finally, we broadened searches 1 and 2 to include any health-related initiatives (not just tobacco) addressing communities and convergence, using the following search string:

Search 3: health AND (collaboration OR partnership) AND (community OR state OR city OR county) AND convergence*.
Abstracts were assessed for relevance to multisectoral collaboration and convergence in health-related initiatives broadly or tobacco control specifically. All abstracts were appraised by two authors to determine eligibility for inclusion. Disagreements were resolved after consultation between the two appraisers and an additional author. Relevant articles were synthesized to glean a common framework and are discussed and presented in narrative form.

\section{Results}

The initial search (community tobacco programs involving collaboration or partnership) resulted in 134 results. The second search (expanding search 1 to include specifically convergence or innovation) returned three results. The final search (geographic-based community health initiatives in any public health area involving convergence) returned 43 results.

Only studies reflective of multisectoral collaboration in a specified geographical location and/or tobacco control were included for in-depth review. Out of the original 180 articles, after removing duplicates, 23 met the criteria for final inclusion (Table 1).

\section{Discussion}

While complete convergence was sparse, we identified numerous initiatives and programs that applied multisector collaboration in the planning and implementation of public health programs. These studies displayed an interesting array of convergence applications. They varied in the number of partners, ranging from two to including all participants of a society, ${ }^{30}$ specialty (mental health, tobacco control, substance abuse, suicide, and obesity), systemic/ geographical reach (university system, health care system, state, and global system), and types of communities (e.g., neighborhoods, towns, cities, and states). Other models of collaboration appeared as examples of convergence. Three studies included Community-Based Participatory Research (CBPR $)^{31-33}$ as a model for collaboration, one study described Practice-Oriented Research (POR), ${ }^{34}$ and one study looked at the Collective Impact model. ${ }^{35}$

The articles reviewed enable a comprehensive understanding of applied convergence, its facilitating elements, and the challenges faced during implementation of programs, in which multiple partners and sectors were engaged. Convergent innovation as operationalized in one or more of the following five building blocks provided a useful framework to describe the results: social process innovation, institutional innovation, organizational innovation, technological innovation, and financial innovation. ${ }^{18}$ Within these essential building blocks, 
Table I Summary of literature reviewed

\begin{tabular}{|c|c|c|}
\hline No. & Study & Lessons learned: barriers and facilitators of convergent innovation \\
\hline $\mathrm{I}$ & Bresnahan et $\mathrm{a}^{50}$ & $\begin{array}{l}\text { - Carefully navigate scope and geographical breadth of university systems. } \\
\text { - Apply these six practical steps (Glassman et al) }{ }^{58} \text { aid development and planning: I) create a committee to drive } \\
\text { the process, 2) develop committee initiatives, 3) allow student debate of proposed changes to existing policy, 4) } \\
\text { generate publicity, 5) draft potential policy, and 6) focus communication efforts on the Board of Trustees. } \\
\text { - Consider campus-wide tobacco policy working groups and involvement of students. } \\
\text { - Create culture of compliance. } \\
\text { - Train clinic personnel on tobacco counseling. } \\
\text { - Involve human resources to disseminate policy to staff and generate staff support. } \\
\text { - Use multiple communications strategies for publicity and dissemination. } \\
\text { - Garner resources to support implementation. }\end{array}$ \\
\hline 2 & Blanchard et al ${ }^{49}$ & $\begin{array}{l}\text { - Monitor changes in leadership support and personnel to avoid stalled progress. } \\
\text { - Anticipate policy and regulatory changes, such as economic impact from tobacco bans. } \\
\text { - Consider changing political climates that may affect the partnership. } \\
\text { - Assess ability of non-native researchers (noncommunity members) to broach sensitive Tribal (community) } \\
\text { concerns. } \\
\text { - Get clarity on partners' commitment to extended periods of collaboration. } \\
\text { - Take time for trust building between researchers and Tribal partners and other community members. } \\
\text { - Ensure collective ownership, beginning with the actual planning process. }\end{array}$ \\
\hline 3 & Castonguay et $\mathrm{al}^{34}$ & $\begin{array}{l}\text { - Anticipate difficulties in collecting meaningful data on multiple levels and multiple phases. } \\
\text { - Determine circumstances or activities perceived as irrelevant or detrimental to stakeholder groups. } \\
\text { - Understand differing vernacular language - stakeholders frequently "talk different languages". } \\
\text { - Consider issues of feasibility - research protocols that require too many tasks or intense supervision are } \\
\text { difficult to implement. } \\
\text { - Anticipate communication problems that could jeopardize design, planning and implementation, and trust. } \\
\text { - With many stakeholders involved, carefully orchestrate the exchange of information. } \\
\text { - Consider interpersonal dynamics as potentially challenging. } \\
\text { - Consider stakeholder incentives, financial, and otherwise. } \\
\text { - Keep the research relevant - to generate and maintain practitioners' commitment, studies have to go beyond } \\
\text { the threshold of "clinical relevance" and address the ways of improving clinical practice. } \\
\text { - Address stakeholder threats and anxiety through transparency. } \\
\text { - Consistently gather stakeholder feedback - set up feedback loops. } \\
\text { - Develop a "sense of community" that is guided by the shared ambition. } \\
\text { - Utilize technology. }\end{array}$ \\
\hline 4 & Farrington et $\mathrm{a}^{30}$ & $\begin{array}{l}\text { - Engage the target populations early on and throughout implementation. } \\
\text { - Avoid the miscalculation of time needed for negotiation of involvement of partners - incorporate the needs and } \\
\text { interests of multiple stakeholders. } \\
\text { - Employ evaluation at all phases. } \\
\text { - Consider needed resources up front - and revisit to avoid deficits that interfere with mission. } \\
\text { - begider how to maintain momentum over the long run - sustainability of the intervention starts in the } \\
\text { - Know the needs of the target population - ask, do not assume. } \\
\text { - If possible, integrate with a major citywide initiative. } \\
\text { - Employ participation and codesign of all products with multiple stakeholders. } \\
\text { - Involve private companies (if relevant) and media relations from the very beginning. } \\
\text { - Collaborate with research and training institutions, academic and industry affiliated. } \\
\text { - Set clear leadership and governance. } \\
\text { - Consider action and visibility at the national level whenever possible. } \\
\text { - Pursue and lay out possible funding opportunities. }\end{array}$ \\
\hline 5 & Flood et a $\left.\right|^{35}$ & $\begin{array}{l}\text { - Be aware that inadequate funding limits accomplishments. } \\
\text { - Be tuned in to diversity - diversity of Coalition members in income, education, race/ethnicity, age, and resident } \\
\text { versus outsider status can create tensions based on power differentials. } \\
\text { - Consider five core tenets of collective impact: a common agenda, shared measurement, mutually reinforcing } \\
\text { activities, continuous communication, and backbone organization. } \\
\text { - Know the varying landscapes of other key players - within the geographic communities and the affiliated agencies. } \\
\text { - Engage public-private partnership, including community members, organizations, government officials, and } \\
\text { for-profit entities. } \\
\text { - Engage/employ an influential champion connected to the community or problem of interest. }\end{array}$ \\
\hline
\end{tabular}


Table I (Continued)

\begin{tabular}{|c|c|c|}
\hline No. & Study & Lessons learned: barriers and facilitators of convergent innovation \\
\hline 6 & Leuthard et al $^{46}$ & $\begin{array}{l}\text { - Integrate with system within health care settings - in this case groups partnered with statewide health care } \\
\text { systems to integrate helpline referrals into regular patient care. } \\
\text { - Build strong partnerships with care providers. } \\
\text { - Apply technological innovation. } \\
\text { - Train health providers in relevant topics - in this case they were educated about the evidence-based 5A's (Ask, } \\
\text { Advise, Assess, Assist, and Arrange) tobacco cessation protocol. }\end{array}$ \\
\hline 7 & Momin et $\mathrm{al}^{42}$ & $\begin{array}{l}\text { - Beware of high staff turnover, leading to loss of institutional memory. Turnover made it difficult to know } \\
\text { partners, thereby decreasing staff engagement in coprogram efforts. }\end{array}$ \\
\hline 8 & Rhoades and Beebe ${ }^{44}$ & $\begin{array}{l}\text { - Know the implications of potential or anticipated tobacco regulatory changes over time. } \\
\text { - Partnerships eliminate duplication and ensure efficient use of public health dollars. } \\
\text { - Accept that community coalitions can operate successfully within larger convergent efforts. } \\
\text { - Leverage the unique attributes of partners, efficient use of resources, and prioritizing systems and communities } \\
\text { as key agents. } \\
\text { - Integrate tobacco initiatives into larger wellness approaches within the targeted community. } \\
\text { - Consider comprehensive media campaigns and consistent public relations. }\end{array}$ \\
\hline 9 & Sarin et $\mathrm{al}^{21}$ & $\begin{array}{l}\text { - Embrace leadership through partnership. Working in aboriginal community controlled health services } \\
\text { (ACCHSs), Local Health Districts, and other stakeholder organizations from rural and urban areas. } \\
\text { - Embrace community action, awareness, and engagement. } \\
\text { - Increase the capacity of native (Aboriginal) communities and workforce development. } \\
\text { - Foster a supportive environment. } \\
\text { - Improve access to nicotine replacement therapy and other medications to support quitting. } \\
\text { - Coordinate and integrate efforts to address smoking in the general population, and within other Aboriginal } \\
\text { health programs and activities. }\end{array}$ \\
\hline 10 & Tong et $\mathrm{al}^{39}$ & $\begin{array}{l}\text { - Collaborate with partners to develop set of core evaluation measures that are available on an interactive platform. } \\
\text { - Foster cultural competence as essential across partners. } \\
\text { - Create partnerships between statewide and nationwide funding organization facilitate generation of ideas, } \\
\text { - Allow flexibility in networks to design and implement strategies to reflect the specific context and conditions of } \\
\text { the targeted communities. } \\
\text { - Use social and community networks to the fullest extent possible. } \\
\text { - Train partners to build capacity and synergy for community-based research. }\end{array}$ \\
\hline II & Allen et $\mathrm{al}^{31}$ & $\begin{array}{l}\text { - Consider extreme diversity of cultural groups within American Indian/Alaska Natives populations. } \\
\text { - Understand that lack of trust stalls progress and trust must be employed at all phases. } \\
\text { - Assess methodological challenges including the costs of working in remote, geographically dispersed settings. } \\
\text { - Employ cultural understanding including local cultural practices for conducting activities and identifying and } \\
\text { respecting leadership. Interventions must fit with community values and traditions. } \\
\text { - Build on protective factors existing in community; do not highlight differences or problems as inherent to the } \\
\text { community. }\end{array}$ \\
\hline 12 & Díaz-Toro et $\mathrm{al}^{36}$ & $\begin{array}{l}\text { - Consider political instability. } \\
\text { - Anticipate economic crises. } \\
\text { - Carefully consider time constraints of various partners. } \\
\text { - Understand that tobacco control has many competing health priorities within communities and organizations. } \\
\text { - Address lack of professional competence in tobacco-related topics. } \\
\text { - Build capacity of network members. }\end{array}$ \\
\hline 13 & Mcneill et $\mathrm{al}^{37}$ & $\begin{array}{l}\text { - With intelligence sharing, assume different philosophies among agencies and a lack of trust between some } \\
\text { stakeholders; conversely, competing values can improve the flow of intelligence. } \\
\text { - Carefully weigh how lack of trust, different structures, and different cultures can be limiting and time consuming. } \\
\text { - Recognize the importance of differences in roles across members and partner groups. }\end{array}$ \\
\hline 14 & Moody-Thomas et al ${ }^{59}$ & $\begin{array}{l}\text { - Address competing priorities among stakeholders. } \\
\text { - Establish systems to connect and communicate across stakeholders, including the community. } \\
\text { - Train members and personnel to interact with the priority population - cultural competence is critical. } \\
\text { - Help administration and staff understand why tobacco control should be a priority. }\end{array}$ \\
\hline 15 & Mendenhall et $\mathrm{al}^{33}$ & $\begin{array}{l}\text { - Avoid exhaustion of funds by tapping into extant community resources and energies. } \\
\text { - Find equity in project ownership to bring additional resources from each partner. } \\
\text { - Channel capacity of ordinary people as producers of health and social change for themselves and their } \\
\text { communities. } \\
\text { - Spin initiative as a social movement. }\end{array}$ \\
\hline
\end{tabular}


Table I (Continued)

\begin{tabular}{|c|c|c|}
\hline No. & Study & Lessons learned: barriers and facilitators of convergent innovation \\
\hline$\overline{16}$ & Schoen et al ${ }^{54}$ & $\begin{array}{l}\text { - Network metrics such as degree, density, and centralization can describe relationships among people and } \\
\text { organizations and can reveal differences in communication and collaboration among coalitions. } \\
\text { - Evaluate the process of converging programs and measure the impact of partnership community capacity. } \\
\text { - Incorporate community capacity building. }\end{array}$ \\
\hline 17 & Dubé et al ${ }^{18}$ & $\begin{array}{l}\text { - Build partnerships. } \\
\text { - Social learning process can be challenging when partners who have been traditional competitors learn how to } \\
\text { recognize and embrace the value proposition of potential collaborators. } \\
\text { - Develop a universal blueprint for deploying initiatives. } \\
\text { - Strategically engage private enterprises. } \\
\text { - Determine the optimal ways to mobilize the community and address needs of the most underserved. } \\
\text { - Apply technological innovation, social process innovation, organizational innovation, financial innovation, and } \\
\text { institutional innovation. } \\
\text { - Other factors to consider: composition of consortia, partners' approach to innovation, experience with } \\
\text { collective action, capacity to act together, careful design of consortium, presence of visionary and powerful } \\
\text { catalysts, grass roots efforts, leadership, metrics. }\end{array}$ \\
\hline 18 & Ekwenugo et $\mathrm{a}^{45}$ & $\begin{array}{l}\text { - Employ crosscutting collaboration of members with state health departments, cancer control coalitions, and } \\
\text { other community-based groups. } \\
\text { - Determine different partner roles and assets (e.g., raise awareness, provide education, leadership guidance, } \\
\text { technical assistance, and so on). } \\
\text { - Use technological innovations: one of the Cancer Prevention and Control Research Network (CPCRN) } \\
\text { intervention tools, Make It Your Own (MIYO), is a Web-based, interactive social media system, allowing the } \\
\text { user to select from evidence-based approaches such as small media and client reminders. }\end{array}$ \\
\hline 19 & Gehlert $^{38}$ & $\begin{array}{l}\text { - Noncommunication between disciplines results in research-based recommendations seldom being coordinated. } \\
\text { - Reward investigators for discovering and for working collaboratively - lack of a reward system may result in } \\
\text { failure to adequately inform health care providers, the public, and policy-makers about the work. } \\
\text { - Employ universities and other research institutions to set up a relevant education, training, or curriculum to } \\
\text { teach transdisciplinary research as early in the educational process as possible - train the next generation. } \\
\text { - Find ways for members to acknowledge the other members' contributions and respect the cultures of other } \\
\text { members' disciplines. }\end{array}$ \\
\hline 20 & Allen et $a^{160}$ & $\begin{array}{l}\text { - Apply community-based participatory research (CBPR) approaches, which recognize the strengths, knowledge, } \\
\text { expertise, and resources of communities, and engage community members in the research process as full } \\
\text { partners, increasing the relevance, feasibility, and cultural appropriateness of interventions. }\end{array}$ \\
\hline 21 & Healton and Curan ${ }^{61}$ & - Increase the capacity of communities to tackle the tobacco issue head on. \\
\hline 22 & Macaller et al ${ }^{62}$ & $\begin{array}{l}\text { - Enlist the support of existing, committed, and trusted health care providers to serve as cessation promoters or } \\
\text { interventionists. }\end{array}$ \\
\hline 23 & Wynn et $\mathrm{a}^{32}$ & $\begin{array}{l}\text { - Apply the principles of CBPR. } \\
\text { - Utilize lay volunteers - volunteers held in high regard in their communities as natural helpers. } \\
\text { - Apply grass roots organizing strategies. } \\
\text { - Consider an innovation spiral - identify other (related) issues that can benefit from similar action steps and } \\
\text { strategies (e.g., tobacco legislation, breast, and cervical cancer). }\end{array}$ \\
\hline
\end{tabular}

our review highlighted the following three common themes: collaboration, community mobilization, and leadership.

\section{Social processes}

Collaboration underlying convergence is a social process innovation as it changes the ways in which individuals interact and creates new opportunities and activities. Collaboration among multisector organizations is essential in addressing the gaps in tobacco control and other public health issues. ${ }^{36}$ The absence of collaboration can constrict the reach and impact of public health initiatives. For instance, barriers to enforcement of illicit tobacco trade emerge from a lack of partnership and trust between different organizations and can be overcome by partnerships between health, marketing, and enforcement agencies. ${ }^{37}$ As suggested by Gehlert, ${ }^{38}$ collaboration is ideal when it is both transdisciplinary and translational. Partners need to collaborate at every level: knowledge synthesis, initiative design, implementation, and dissemination. ${ }^{38}$ Such codevelopmental collaboration, especially by community representation and coalitions, is attested by numerous other studies. $^{21,30,31}$

Farrington et $\mathrm{al}^{30}$ describe a large evaluation of the WHO European Healthy Cities Network during its fifth phase (2009-2013), which focused on the prevention of four behavioral risk factors: tobacco use, alcohol abuse, unhealthy diet, and physical inactivity. The evaluation identified multisector collaboration and partnership as a facilitator of prevention program success. Successful programs also featured 
leadership, community involvement, involvement of private enterprise, and equity. ${ }^{30,36,39}$ Partnerships with communities can directly address health inequities (beyond the impact of initiatives) by promoting social cohesion and increasing a sense of community identity. ${ }^{30}$ Of note in the Healthy Cities Network, the proportion of partnerships was highest among tobacco control programs in comparison to food-related and alcohol/drug-related programs.

Similarly, Tong et $\mathrm{l}^{39}$ mention how partnerships between statewide and nationwide organizations facilitate not just synchronous and more impactful implementation activity but also are essential for idea generation, recruitment, and organization. Partnerships provide a level of flexibility that allows different sectors to identify and implement strategies relevant to the context and conditions of communities. ${ }^{39}$ Partnerships also bring access to much needed resources by leveraging the different attributes of members. For maximum utility, these partnerships need to be crafted early at the knowledge synthesis phase, where ideas and strategies are generated and agendas can be set. ${ }^{35}$ That is the only way in which disparate groups can gain a holistic picture of the issue, its determinants, and speak to the myriad strategies suitable to addressing it. ${ }^{38}$

Adopting a systems approach is more challenging in the real world than in concept, especially when collaborators are used to working and thinking in silos. Assumptions on dos and do nots for the private and nonprofit world can create tension. A social learning phase is essential for collaborators who speak and think in discrepant languages and roles to understand the "value proposition"18 each brings to the playing field. The playing field itself does not have to be "captured" by the private sector nor does it have to be "leveled" by nonprofits; instead partners work together to "raise" it. ${ }^{18}$ Removing mental blocks can be challenging and is an ongoing process to create the collaborative effervescence that has come to describe successful collaborations. ${ }^{40}$ The goal surrounding convergent innovation - to respond to the community's need and to focus on the most underserved - itself has the ability to organize and unify around common empathy and a higher cause. The challenge comes in recognizing useful differences among partners in order to leverage them. The emotional connectivity ${ }^{41}$ and collective cognition required to foster convergence results in a culture change that initiates in the collaborative consortium and spills over into the specific community whose needs are being addressed. This can be facilitated by the strategic integration of individual initiatives at the conception phase of a program or through codevelopment, where partners are engaged in the program design phase or even earlier as part of knowledge development preceding the design phase.

Without strategic and ongoing activities that enhance collaboration, partnerships are susceptible to numerous organizational, logistic, interpersonal, and cultural challenges. ${ }^{34,42}$ A roadmap for the process of convergence innovation is a useful guide for partnership development in three iterative phases: social learning, social capital formation, and collective action. ${ }^{18}$ Since each collaborator "speaks in a different language", ${ }^{43}$ a social learning phase is an essential stage for all partners to learn a common language and bridge differences while collectively leveraging their attributes ${ }^{34,44}$ and perspectives. It is also an opportunity for capacity building in knowledge, resources, technology, infrastructure, or organizational processes. ${ }^{21}$ Capacity building is cited in numerous studies as a strong catalyst for partnerships as well as for community mobilization and empowerment..$^{33,36,45}$ The social learning phase strengthens the social capital that exists in the form of these partnerships. It also creates opportunities to address partner threats and anxiety through transparency. ${ }^{34}$

Among partners, some may be more distinguished than others. The inclusion of private enterprise as a partner may be critical to innovation as it relates to technologically advanced resources. Since the community is considered the primary recipient of the convergent innovation, representatives from the communities act both as partners and as key change agents.

\section{Organizational and institutional processes}

As both the agent influencing and experiencing the issues at hand, the community becomes a partner and the beneficiary of a convergent initiative. Communities therefore represent a form of institutional innovation and are mentioned in nearly all articles reviewed as significant contributors to any collaborative initiative. Experience with collective action is also beneficial as is the inclusion of clinical providers within prevention programs. ${ }^{34,46}$ Numerous studies mention community mobilization as a means to empower communities to impact social and health issues as well as an outcome of multisector collaboration. ${ }^{30-33,35,36,44,45}$ It is a key enabler of convergent innovation especially in a technologically-savvy world where Internet and mobile connectivity facilitate better and quicker organization of communities. ${ }^{18}$ Communities are best aware of their needs as well as their strengths, and once engaged, resourced, and mobilized, they can become an impetus for sustained convergent innovation and impact. ${ }^{18,32}$ The analysis of a cross-sector collaboration using models of community mobilization for large-scale social change (Collective Impact 
model and Community Coalition Action Theory) by Flood et $\mathrm{a}^{35}$ illustrates the five core tenets of Collective Impact: shared agenda, shared measurement systems, mutually reinforcing activities, continuous communication, and central infrastructure. The Collective Impact model is consistent with convergent innovation principles in aligning multisector partners, especially Chief Executive Officers of nonprofit organizations with private business leaders and funders to eliminate duplication of efforts while enhancing impact. ${ }^{47,48}$ Developing a nonexploitive relationship with community members based on trust and cultural understanding is a precept to community engagement and mobilization. . $^{31,49}$

In light of the multitude of organizational barriers to convergence evident in our review, organizational innovation is a significant means of attaining successful convergence. From coordinating the multiple partners and incorporating their needs and interests, program implementation, hiring, and retaining staff (in an area where staff turnover is high) to navigating the logistical and communication challenges of disparate infrastructure and administrative operations, this is a territory that needs considerable business process expertise, management prowess, and leadership skills. Orchestrating the exchange of information in a beneficial manner, maintaining camaraderie, and minimizing conflict are primary roles of the organizational leadership.

While it may be obvious that leadership is vital for the success of any large-scale enterprise, several studies consider it a decisive factor in the success or failure of convergence. In a qualitative study on collaboration between national cancer control and tobacco control programs, Momin et $\mathrm{al}^{42}$ emphasize that leaders are the ones who need to define collaboration and create guidelines for partners to work. They engage decision makers and stakeholders across sectors and are able to convey the sense of urgency and sense of camaraderie crucial to create and sustain partnerships. Leadership not only is a catalyst for relationship building within collaborations but also provides visionary guidance on the organizational, financial, and technical fronts. Leaders are responsible for the organizing principles of convergent innovation such as management, infrastructure, and recruitment. Tong et $\mathrm{al}^{39}$ recommend that leadership must collaborate with partners to develop appropriate evaluation measures in order to track progress. Lack of leadership is a barrier to collaboration as are discrepant models of leadership among collaborators. ${ }^{42}$

\section{Financial processes}

The influence an organization can wield on the consortia members is limited by each member's "voluntary" commitment. Organizational innovation thereby will eventually need an appropriate financial process in order to incentivize commitment and establish accountability. As revealed by the review, innovation in financial processes is incipient at best. Current funding requirements hinder collaborators from sharing resources and integrating interventions. ${ }^{42}$ Most collaboration is still contingent upon traditional grant/allocation funding based on federal and state funding requirements. Funding limitations can be circumvented by utilizing existing community facilities, employing community members, and sharing collaborator resources to minimize costs as well as assist in program implementation. ${ }^{35}$ For instance, Bresnahan et $\mathrm{al}^{50}$ describe a successful partnership between the NY Health Department and CUNY university, which was supported considerably by the provision of resources and technical assistance by the former. Innovative financial processes need to be appropriately developed in order to adequately incentivize and sustain convergence. Codevelopment of programs can allow for joint grant writing and noncompetitive funding streams for the collaborative effort. The ability of a program's funding to serve for another program's benefit, or mutual funding, is described in the study by Momin et $\mathrm{al}^{42}$ as a facilitator for collaboration.

\section{Technological processes}

Likewise, a convergent innovation cannot thrive without technological innovation, and in many instances, technological advances have spurred collaborations. ${ }^{51}$ As described by Leuthard et $\mathrm{al}^{46}$ and Castonguay et al, ${ }^{34}$ technological processes can improve communication channels between collaborators and the actual innovation product. A simple system-wide technological solution to connect tobacco users to a helpline using fax referrals created the clarity and direct timely communication that was critical to the success of the initiative and strength of the stakeholders. ${ }^{46}$ This is especially true in the present day, where technological processes go beyond just expanding access and reach to the community; they create ease in communications and bring the reciprocity through which the people come to the program instead of just taking the program to the people. Technology is key to synchronizing programmatic activities ranging from the integration of real time information to giving marketing facelifts to campaigns and health communication materials. One example of a technological convergence innovation is community-based innovation using collaborative online innovative networks (COINS).$^{52}$ This model of convergence resides on Internet-based and mobile communication networks, where communities of interest include partners from 
multiple disciplines and sectors. ${ }^{53}$ The online models are realized as face-to-face interactions at the local level.

One of the challenges in measuring success across networks is a lack of established common factors. ${ }^{39,54}$ Technological innovations need to include new ways of collecting data at multiple levels by multiple stakeholders at numerous points of impact. The lack of accompanying evaluation and quality metrics may render some convergent innovations unmeasurable and undermine any impact. Moreover, on the systemic level at which convergence works, there is a multiplicity of overlapping effects. Traditional process and outcome evaluations may need to be modified and adapted for intramodular and intermodular assessment using unique models to measure convergence. Intermodular evaluation will require the development of evaluation measures that include measures of collaboration such as network metrics. ${ }^{54}$ Composite measures and indices of convergence elements may also need to be developed and tested.

\section{Case example}

The DC Metro Tobacco Research and Instruction Consortium (MeTRIC) is exemplary of emerging convergent innovation for tobacco control. Located in the policy and political hub of the US (Washington, DC), MeTRIC was born out of a growing need to address persistent tobacco-related health inequities in the DC metroregion. DC, plagued with extraordinarily high tobacco rates in its most diverse neighborhoods, is also home to some of the top tobacco control scientists in the world and a dedicated cadre of health officials. Yet the thread linking the problem to the full complement of problem solvers was not fully woven. Chartered in spring 2013, MeTRIC formed as a consortium of regional experts and advocates uniquely positioned to respond to the tobacco-related community health needs of this urban setting. Researchers from two academic institutions - Milken Institute School of Public Health at George Washington University, Georgetown University - and The Schroeder Institute at the Truth Initiative, which is the largest US nonprofit organization dedicated to tobacco control, are at the core of the consortium. Students from these institutions, alongside community advocates, practitioners, and Department of Health officials are also engaged as members and partners. By converging these stakeholders and their diverse levels of knowledge, expertise, and influence, MeTRIC functions as a vehicle for tobacco control research, dissemination, and implementation of educational activities. The mission of MeTRIC is to, 1) develop and conduct community-driven multilevel research that reduces tobacco use among those most impacted by health disparities in the DC metroarea; 2) foster collaborations with medical, public health, and community partners to translate research findings into culturally competent tobacco-related policy and practice in the DC metroarea; 3) implement a training program to prepare the next generation of tobacco control researchers; and 4) provide leadership to local, state, and federal government in the advancement of evidence-based policy and practice, beginning in DC (http://publichealth. gwu.edu/projects/metric).

MeTRIC bypasses some of the challenges and pitfalls of prior collaborative approaches through convergence. The consortium aspires to reduce problems associated with largescale initiatives in complex urban cities such as DC. Bringing together the right complement of partners is intended to reduce the challenges resulting from implementation of tobacco control tools and programs that lack meaning and value to the communities; lack of broad expertise needed to implement a range of tobacco control approaches; lack of expertise and buy-in to obtain grants; insufficient resources, including human capital for research and training; lack of leadership at multiple levels; and lack of ongoing feedback loops, including inadequate evaluation. Through the engagement of important health officials and policy-makers, convergence will also enhance the likelihood that MeTRIC efforts have sustainability over time through policy and practice changes. Although in its infancy, MeTRIC is engaged in social, organizational/institutional, and financial innovation processes, which are the building blocks of convergence. ${ }^{18}$ A few examples are presented later.

\section{Social process innovation}

DC is a complex community, with many organizations focused nationally rather than locally. Interactions among key constituents around tobacco control in DC were not common. One of the biggest challenges was changing the way individuals and organizations that work in tobacco control in the DC metropolitan region interact with each other. MeTRIC creates a platform in which researchers and community groups can cross paths and share knowledge toward reducing tobacco use in the DC area. The consortium affords researchers and community advocates ways to conduct research together outside of their own institutions (e.g., through pilot grant programs, joint training ventures, and joint retreats). For instance, new electronic communication platforms (e.g., LISTSERVS, Web site, and shared databases) created by MeTRIC that cross institutions have created a virtual community on the basis of a shared dedication to decreasing tobacco-related disparities, while regular in-person convening is forming a "real-world" community of consortium members. These interactions open up new opportunities to MeTRIC members as well as to the entities they work for and 
with. Community advocates and practitioners have access to experts in cessation, health care, behavior change, and policy, while academics and other scientists have the opportunity to engage practitioners about what science is needed to inform policy and practice. To enable better service and value across the consortium, future communications platforms will be developed to enable faster and more collaborative exchanges of information, such as through social media, apps, and highly interactive Web presence for members.

\section{Organizational and institutional innovation}

Organizationally, MeTRIC has created a cross-institution consortium of experts, students, and community members with a passion for tobacco control. To overcome the challenges of institutional complexities and fixed ways of doing business, we established new interorganizational structures and new processes of working together for mutually serving goals. For instance, the founding institutions are dedicated to training the next generation of tobacco control professionals, and through MeTRIC we now have an exchange system in place for students to complete their required practicum or other training requirements under the guidance of a MeTRIC member scientist at different institutions. This does not only fulfill MeTRIC goals but also maintains stakeholder interest and strengthens partnerships. Another key innovation is an academic course developed by MeTRIC (course on the fundamentals of tobacco-related health inequities) that will soon be available not only to the member institutions but also to students enrolled at any of the 14 member institutions making up the Consortium of Universities of the Washington Metropolitan Area. ${ }^{55}$ Showing community buy-in, health department officials have expressed interest in practitioners auditing the course. To avoid duplication and optimize productivity, MeTRIC members share resources - each stakeholder not only brings his or her expertise to the group but also shares data, methods, laboratories, and technology with all members of the consortium. While MeTRIC is operationally housed at one of the three founding institutions (George Washington University), each of the three institutions is represented by a codirector. The codirectors serve as the guiding leadership team. Though competition was not a problem we encountered among our founding institutions, a shared leadership model can minimize that possibility. It is intended to maintain high participation, heterogeneity of expertise, and strong mutual interest among key partners.

\section{Financial innovation}

MeTRIC's greatest challenge is fiscal sustainability. The full financial structure of MeTRIC is not yet realized but there is mutual commitment to grow our financial viability. In turn, the consortium is pursuing joint funding mechanisms. One way MeTRIC exemplifies convergent innovation is through cross-institutional funding. Having realized that a lack of financial incentive poses a barrier to convergent efforts following MeTRIC retreats, MeTRIC recently initiated a small pilot grant program to foster innovative team science across MeTRIC members. The purpose of the pilot grants, which originate from GW Milken Institute of Public Health internal funds, is to jump start collaborative studies across identified thematic areas that increase likelihood of federal funding for multidisciplinary research in the future. Awardees are required to include investigators from all three primary partners, include at least one community partner, and at least one graduate student affiliated with the primary partner organizations. Team member institutions agreed to support the dedicated efforts of the individuals involved in the projects. Essentially, all institutions have a financial stake in the pilot program. With an emphasis on shared successes, the joint contract for awards assures that all institutions are represented in resulting products, including grant proposals and publications. In the inaugural pilot program, five interconnected awards were granted (start date August 2016), all focused on tobacco-related priorities in DC.

\section{Recommendations for convergent approaches}

Based on the MeTRIC experience, the work of Dubé et al ${ }^{18}$ and Stillman et $\mathrm{al}^{56}$ and other initiatives reported in the appraised literature, an innovative convergent tobacco control research approach may have the following characteristics:

- Member driven and organized: it relies on the passion of the people to move the work.

- Not-for-profit but inclusive of private enterprise: organizations are designing for the greater good.

- Noncompeting: organizations are able to share freely and plentifully, the best and worst of their individual worlds.

- Friendship oriented: organizations plan events that foster not only knowledge development and exchange, but also deep connections between organizations.

- Product oriented: developed products can be "good enough" along traditional dimensions but should be exceptional along new dimensions.

- Relevant today: public health innovation in real-world settings is context driven and must be meaningful to the experiences of the communities and intended recipients, in real time.

- Forgiving: mistakes are inevitable. Innovation involves a process of trial-and-error in which mistakes are frequent and from which much can be learned. 
- Iterative: feedback is critical and evaluating a convergent innovation requires nontraditional methods - for instance, because it may be difficult to know what aspect of the innovation is influencing change, there may be utility in creating composite or indexed measures of convergence.

- Disruptive: by design, these innovations can be disruptive, should challenge traditional thinking and may provoke controversy.

- Innovation forward: participating organizations can create protective environments, insulating innovation teams from operational pressures; others may create permissive "learning environments" within mainstream structures. There should also be concerted effort across partners to create an environment and culture that supports the innovation partnership.

- Well led: buy-in and support from senior leadership is essential across all converging groups.

- Investment oriented: return on the investment has to be great enough to warrant support of all partners, including community members.

- Sourced: funding streams should be identified for sustainability.

- Fluid: highly evolved systems with deeply entrenched organizational layers of decision making create complexity for the innovative process.

- Reward balanced: clear high-level leadership is necessary but not always well resourced. The administrator of a collaborative project needs to be compensated in some way as he or she is the bridge and communicator between all stakeholders and multiple components of the project.

- Dedicated: members need dedicated work time to focus on the innovation.

- Inclusive: collaboration should comprise members along the research-practice-policy continuum: practitioners and community stakeholders (including patient populations) should collaborate with researchers.

\section{Conclusion}

This literature review gleaned important insights on the nature of convergence and its multiple applications in public health innovations. "Systems thinking" is not new in public health or tobacco control. Collaboration is, to varying degrees, inherent in a variety of approaches, be it in systems thinking, translational research, transdisciplinary research, POR, and CBPR. Convergence is a more expansive concept that encompasses all of this and more. With its two definitive principles in which, 1) public health and economic development go hand in hand, and 2) the focus is on improving the status of the underserved sector of society, convergence leans on innovation to address specific needs of the community. With this goal as a starting place, convergence becomes the natural means for disparate groups within a community to leverage their skills and resources. Our review of the literature revealed that the concept of convergence is being applied to more comprehensively and sustainably address common public health issues. In the multidisciplinary world of tobacco control, the role of convergent innovation in addressing gaps resistant to current efforts shows promise but is not yet fully realized. While researchers, health providers, communities, policy-makers, economists, behavioral scientists, and tobacco users and quitters establish these collaborations, the need for novel organizational, financial, and technological innovations is imperative and required ${ }^{57}$ In the meantime, real-world experiences of convergent innovation consortia such as MeTRIC will reveal important insights to guide in the creation and implementation of a much needed framework for convergence in tobacco control. Convergence innovation thrives on partnerships among those who "dream big", ${ }^{43}$ requiring not only hard work but also "ambitious goals". ${ }^{34}$ It also recognizes "the diversity, complexity, and dynamic nature of conditions and contexts", ${ }^{18}$ which serve as challenges and opportunities to develop a universal process for designing and implementing convergent innovation projects. On the whole, we take away important guidance for formulating and implementing a convergent innovation.

\section{Disclosure}

K Horn, KP Tercyak and R Niaura are Directors of MeTRIC. The authors report no other conflicts of interest in this work.

\section{References}

1. WHO [webpage on the Internet]. WHO Report on the Global Tobacco Epidemic, 2011. World Health Organization; 2013. Available from: http://www.who.int/mediacentre/fs339/en/). factsheets. Accessed July 24, 2016.

2. US Department of Health and Human Services. Executive Summary (The Health Consequences of Smoking - 50 Years of Progress: A Report of the Surgeon General). Bethesda, MD: U.S. Department of Health and Human Services; 2014.

3. Roeseler A, Solomon M, Beatty C, Sipler AM. The tobacco control network's policy readiness and stage of change assessment: what the results suggest for moving tobacco control efforts forward at the state and territorial levels. J Public Health Manag Pract. 2016;22(1):9-19.

4. US Department of Health and Human Services; Centers for Disease Control and Prevention (CDC). Centers for Disease Control and Prevention. Best Practices User Guides-Health Equity in Tobacco Prevention and Control; 2015. Available from: http://www.cdc.gov/tobacco/ stateandcommunity/best-practices-health-equity/pdfs/bp-health-equity. pdf. Accessed July 24, 2016.

5. Warner KE. Disparities in smoking are complicated and consequential. What to do about them? Am J Heal Promot. 2011;25(sp5):S5-S7. 
6. U.S. Department of Health and Human Services National Institutes of Health NCI. Chapter 8, Greater Than the Sum: Systems Thinking in Tobacco Control. Bethesda, MD: U.S. Department of Health and Human Services, National Institutes of Health, National Cancer Institute; 2007.

7. Gottlieb L, Maria Glymour M, Kersten E, et al. Challenges to an integrated population health research agenda: targets, scale, tradeoffs and timing. Soc Sci Med. 2016;150:279-285.

8. Fung M, Simpson S, Packer C. Identification of innovation in public health. J Public Health (Bangkok). 2010;33(1):123-130.

9. Patient Centered Outcomes Research Institute. Transforming PatientCentered Research: Building Partnerships and Promising Models Workshop Report Prepared by Institute for Alternative Futures; 2013. Available from: http://www.pcori.org/sites/default/files/PCORI-Building-Partnerships-Workshop-Report-102712.pdf. Accessed July 24, 2016.

10. National Center for Advancing Translational Sciences NIH [webpage on the Internet]. CTSA: Communities and Research. Available from: http://www.ncats.nih.gov/ctsa/community. Accessed July 22, 2016.

11. Centers for Disease Control and Prevention (CDC) [webpage on the Internet]. Division of Community Heath Programs: Making Healthy Living Easier. Available from: https://www.cdc.gov/nccdphp/dch/ programs/index.htm. Accessed July 20, 2016.

12. National Institutes of Health. Small Business Innovation Research website [homepage on the Internet]. Available from: https://sbir.nih. gov/. Accessed July 20, 2016.

13. Roco MC, Bainbridge WS. Converging technologies for improving human performance: Integrating from the nanoscale. $J$ Nanoparticle Res. 2002;4:281-295.

14. Roco MC, Bainbridge WS. The new world of discovery, invention, and innovation: convergence of knowledge, technology, and society. J Nanoparticle Res. 2013;15:1946.

15. Davis G, Zald M. Social Change, Social Theory, and the Convergence of Movements and Organizations. New York: Cambridge University Press; 2005.

16. Carey G, Malbon E, Carey N, Joyce A, Crammond B, Carey A. Systems science and systems thinking for public health: a systematic review of the field. BMJ Open. 2015;5:e009002.

17. Buckley W. Sociology and Modern Systems Theory. Englewood Cliffs, NJ: Prentice-Hall; 1967.

18. Dubé L, Jha S, Faber A, et al. Convergent innovation for sustainable economic growth and affordable universal health care: Innovating the way we innovate. Ann N Y Acad Sci. 2014;1331:119-141.

19. Bitton A. Moving towards more equitable and integrated approaches for tobacco control and non-communicable diseases: invited commentary. Tob Control. 2012;21:273.

20. Hiscock R, Bauld L, Amos A, Fidler JA, Muna M. Socioeconomic status and smoking: a review. Ann N Y Acad Sci. 2012;1248: $107-123$.

21. Sarin J, Hunt J, Ivers R, Smyth C. Lifting the burden: a coordinated approach to action on Aboriginal tobacco resistance and control in NSW. Public Health Res Pract. 2015;25(3):e2531528.

22. Popay J, Whitehead M, Hunter D. Editorial. J Public Health (Bangkok). 2010;32(2):148-149.

23. Nicholson J. Foreword community health collaboratives: supporting innovation in public policy, care delivery, and coordination. Popul Health Manag. 2013;16(suppl 1):S2-S3.

24. Douglas MR, Manion CA, Hall-Harper VD, Terronez KM, Love CA, Chan A. Case studies from community coalitions: advancing local tobacco control policy in a preemptive state. Am J Prev Med. 2015;48(1S1):S29-S35.

25. Reddy KS, Yadav A, Arora M, Nazar GP. Integrating tobacco control into health and development agendas. Tob Control. 2012;21:281-286.

26. Burch T, Wander N, Collin J. Uneasy money: the Instituto Carlos Slim de la Salud, tobacco philanthropy and conflict of interest in global health. Tob Control. 2010;19(6):e1-e9.

27. Bill and Melinda Gates Foundation. Statement Regarding IDRC Grant: Bill and Melinda Gates Foundation [press release]. Available from: http://www.gatesfoundation.org/Media-Center/Press-Releases/2010/04/ Statement-Regarding-IDRC-Grant. Accessed July 20, 2016.
28. Cohen D. Will industry influence derail UN summit? BMJ. 2011; 343: 55328.

29. Jha SK, Mcdermott J, Bacon G, Lannon C, Joshi PK, Dubé L. Convergent innovation for affordable nutrition, health, and health care: the global pulse roadmap. Ann N Y Acad Sci. 2014;1331:142-156.

30. Farrington JL, Faskunger J, Mackiewicz K. Evaluation of risk factor reduction in a European City Network. Health Promot Int. 2015; 30(S1):i86-i98.

31. Allen J, Mohatt GV, Beehler S, Rowe HL. People awakening: collaborative research to develop cultural strategies for prevention in community intervention. Am J Community Psychol. 2014;54:100-111.

32. Wynn TA, Taylor-Jones MM, Johnson RE, et al. Using community-based participatory approaches to mobilize communities for policy change the collaborative and equitable involvement of various stakeholders in. Fam Community Health Suppl. 2011;1(1S):102-114.

33. Mendenhall TJ, Harper PG, Henn L, Rudser KD, Schoeller BP. Community-based participatory research to decrease smoking prevalence in a high-risk young adult population: an evaluation of the students against nicotine and tobacco addiction (SANTA) project. Am Psychol Assoc. 2013;32(1):78-88.

34. Castonguay LG, Youn SJ, Xiao H, Muran JC, Barber JP. Building clinicians-researchers partnerships: lessons from diverse natural settings and practice-oriented initiatives. Psychother Res. 2015;25(1):166-184.

35. Flood J, Minkler M, Hennessey S, Estrada J, Falbe J. The collective impact model and its potential for health promotion: overview and case study of a healthy retail initiative in San Francisco. Health Educ Behav. 2015;42(5):654-668.

36. Díaz-Toro EC, Fernández ME, Correa-Fernández V, et al. Promoting tobacco cessation and smoke-free workplaces through community outreach partnerships in Puerto Rico. Prog Community Heal Partnersh. 2014;8(2):157-168.

37. Mcneill A, Iringe-Koko B, Bains M, Bauld L, Siggens G, Russell A. Countering the demand for, and supply of, illicit tobacco: an assessment of the "North of England Tackling Illicit Tobacco for Better Health" Programme. Tob Control. 2014;23:e44-e50.

38. Gehlert S. Turning disciplinary knowledge into solutions. $J$ Adolesc Health. 2013;52:S98-S102.

39. Tong EK, Fagan P, Cooper L, et al. Working to eliminate cancer health disparities from tobacco: a review of the National Cancer Institute's community networks program. Nicotine Tob Res. 2015;17:908-923.

40. Boix Mansilla V, Le Lamont M, Sato K. Shared cognitive - emotionalinteractional platforms: markers and conditions for successful interdisciplinary collaborations. Sci Technol Hum Values. 2016;41(4):571-612.

41. Mansilla C, Boix V, Lamont M, Sato K. Successful Interdisciplinary Collaborations: The Contributions of Shared Socio-Emotional-Cognitive Platforms to Interdisciplinary Synthesis. Vancouver, BC: Harvard University; 2012.

42. Momin B, Neri A, Goode SA, et al. Factors involved in the collaboration between the national comprehensive cancer control programs and tobacco control programs: a qualitative study of 6 states, US, 2012. Prev Chronic Dis. 2015;12:E83.

43. Mcaleavey AA, Lockard AJ, Castonguay LG, Hayes JA, Locke BD. Building a practice research network: obstacles faced and lessons learned at the Center for Collegiate Mental Health. Psychother Res. 2015; 25(1):134-151.

44. Rhoades RR, Beebe LA. Tobacco control and prevention in Oklahoma: best practices in a preemptive state. Am J Prev Med. 2015;48(S1):S6-S12.

45. Ekwenugo L, Benard VB, Vinson C. News from the CDC: collaborations to build capacity at the community level in cancer prevention and control. Transl Behav Med. 2013;3:3-5.

46. Leuthard JL, Beebe LA, Halstead L, Olson KD, Roysdon JW. Increased evidence-based tobacco treatment through Oklahoma hospital system changes. Am J Prev Med. 2015;48(1S1):S65-S70.

47. Hanleybrown F, Kania J, Kramer M. Channeling change: making collective impact work. Stanford Soc Innov Rev. 2012:1-9.

48. Kania J, Kramer M. Stanford social innovation review. Stanford Soc Innov Rev. 2011:36-102. 
49. Blanchard JW, Petherick JT, Basara H. Stakeholder engagement: a model for tobacco policy planning in Oklahoma tribal communities. Am J Prev Med. 2015;4(101):S44-S46.

50. Bresnahan MP, Sacks R, Farley S, Mandel-Ricci J, Patterson T, Lamberton P. Going tobacco-free on 24 New York City university campuses: a public agency's partnership with a large urban public university system. J Am Coll Heal. 2016;64(3):343-347.

51. Meyers A. Community based biomedical and health innovation and entrepreneurship and the emerging paradigm of engaging crowds. Technol Transf Entrep. 2014;1(2):82-85.

52. Merkel B. COINs2009: collaborative innovation networks conference COINS: an economic development tool for education, economic and workforce development in Open Source Economic Development. Procedia Soc Behav Sci. 2010;2(2):6516-6531.

53. Gloor PA, Heckman C, Makedon F. Ethical Issues in Collaborative Innovation Networks; 2004. Available from: http://ccs.mit.edu/pgloor papers/coin4ethicomp.pdf. Accessed July 22, 2016.

54. Schoen MW, Moreland-Russell S, Prewitt K, Carothers BJ. Social network analysis of public health programs to measure partnership. Soc Sci Med. 2014;123:90-95.

55. Consortium of Universities of the Washington Metropolitan Area [webpage on the Internet]. Cross-Registration. Available from: http:// www.consortium.org/cross-registration/. Accessed July 20, 2016.
56. Stillman FA, Schmitt CL, Rosas SR. Opportunity for collaboration: a conceptual model of success in tobacco control and cancer prevention. Prev Chronic Dis. 2012;9:E02.

57. Frieden T. Tobacco control progress and potential. JAMA. 2014;311(2): 133-134.

58. Glassman TJ, Reindl DM, Whewell AT. Strategies for Implementing a Tobacco-Free Campus Policy. J Am Coll Health. 2011;59(8): 764-768.

59. Moody-Thomas S, Sparks M, Hamasaka L, Ross-Viles S, Bullock A. The head start tobacco cessation initiative: using systems change to support staff identification and intervention for tobacco use in low-income families. J Community Health. 2014;39:646-652.

60. Allen ML, Garcia-Huidobro D, Hurtado GA, et al. Immigrant family skills-building to prevent tobacco use in Latino youth: study protocol for a community-based participatory randomized controlled trial. Trials. 2012;13(1):242.

61. Healton C, Curan J. The tobacco technical assistance consortium: a foundation - university partnership to reduce tobacco use. Health Promot Pract. 2011;12(S2):112-113.

62. Macaller T, Brown M, Black K, Greenwood D. Collaborating with diabetes educators to promote smoking cessation for people with diabetes: the California experience. Diabetes Educ. 2011;37(5): 625-632.
Innovation and Entrepreneurship in Health

\section{Publish your work in this journal}

Innovation and Entrepreneurship in Health is an international, peer reviewed, open access journal publishing original research, reports, reviews and commentaries on innovation and entrepreneurship in health. Special focus will be given to the theory, process, and practice of innovation and entrepreneurship by individuals and organizations

\section{Dovepress}

within the health care context globally. The manuscript management system is completely online and includes a very quick and fair peer review system, which is all easy to use. Visit http://www.dovepress.com testimonials.php to read real quotes from published authors. 